\title{
Measurements of Bose-Einstein correlations with the ATLAS detector
}

\author{
Ivan Sykora ${ }^{1}$ \\ Department of Nuclear Physics and Biophysics, Comenius University Bratislava \\ Mlynska dolina F1, 84248 Bratislava, Slovakia \\ E-mail: ivan. sykora@emph.uniba.sk
}

on behalf of the ATLAS collaboration

The Bose-Einstein correlations provide a unique opportunity for detailed understanding of space-time characteristics of the hadronization region, for determining the size and shape of the source from which particles are emitted and for interpreting quark confinement effects. The correlations lead to enhancement in production of identical bosons that are close in phase space. The ATLAS collaboration has performed a measurement of Bose-Einstein correlations of pairs of charged particles with transverse momentum greater than $100 \mathrm{MeV}$ in p-p collisions at 900 $\mathrm{GeV}$ and $7 \mathrm{TeV}$. Bose-Einstein correlation parameters are investigated up to very high chargedparticle multiplicities. The dependence of the Bose-Einstein correlation parameters on the average transverse momentum per pair is also investigated.

XXIII International Workshop on Deep-Inelastic Scattering

27 April - May 12015

Dallas, Texas

${ }^{1}$ Speaker 


\section{Introduction}

Bose-Einstein correlations (BEC) are a well-known phenomenon in high-energy and nuclear physics. This effect, as a consequence of symmetry of identical boson wave function, can describe the interference of identical bosons emitted from a localized source. They represent a sensitive probe of the space-time characteristics of the hadronization region and allow the determination of the size and shape of the source from which particles are emitted [1].

Studies of the BEC dependence on particle multiplicity and transverse momentum are of special interest. They help to understand the multiparticle production mechanism. The size of the source emitting the correlated particles has been observed to increase with particle multiplicity. This can be understood as arising from the increase in the initial geometrical region of overlap of the colliding objects, a large overlap implying a large multiplicity.

\section{Bose-Einstein correlations}

The ATLAS detector [2], used for our measurements, is a multi-purpose particle physics device operating at one of the beam interaction points of the LHC. The detector covers almost the whole solid angle around the collision point with layers of tracking detectors, calorimeters and muon chambers. It is designed to study a wide range of physics topics at LHC energies. For the measurements presented in this paper, the tracking devices and the trigger system are of particular importance.

\subsection{Two particle correlation function}

Bose-Einstein correlations are measured in terms of a two-particle correlation function

$$
C_{2}(q)=\frac{P\left(p_{1}, p_{2}\right)}{P\left(p_{1}\right) P\left(p_{2}\right)} \quad ; \quad q=p_{1}-p_{2}
$$

where $P\left(p_{1}, p_{2}\right)$ is the probability to observe two particles with momenta $p_{1}$ and $p_{2}$ and $P\left(p_{1}\right)$, $P\left(p_{2}\right)$ are probability to observe one particle with momenta $p_{1}$ or $p_{2}$

The BEC effect is usually described by a function with two parameters: the effective radius parameter $R$ and the strength parameter $\lambda$, where the latter is also called the incoherence or chaoticity parameter. A typical functional form is

$$
C_{2}(Q)=\frac{\rho(Q)}{\rho_{0}(Q)}=C_{0}[1+\Omega(\lambda, Q R)](1+\varepsilon Q) \quad ; \quad Q=\sqrt{\left|p_{1}-p_{2}\right|^{2}} .
$$

In a simplied scheme for fully coherent emission of identical bosons $\lambda=0$, while for incoherent (chaotic) emission, $\lambda=1, \rho_{0}$ is a two-particle density function (known as the reference function). Experimentally the $\mathrm{C}_{2}$ correlation function is defined as follows:

$$
C_{2}(Q)=\frac{N^{\mathrm{LS}}(Q)}{N^{\mathrm{ref}}(Q)}
$$

Hence it is a ratio of the like-sign (LS) particle pairs $Q$ distribution - signal distributions $N(Q)$ with BEC and reference $Q$ distribution, i.e. particle pairs with no BEC. A good choice of the reference sample is critical for the experimental extraction of the BEC signal. Ideally, $\rho_{0}(Q)$ should include all relevant momentum correlations, from both the local and global conservation laws present in $N^{\mathrm{LS}}$. Several different choices have been studied to construct an appropriate reference sample. The ATLAS analysis is based on use of the unlike-sign particle pairs from the 
same events that are used to form pairs of like-sign particles, i.e., $\rho_{0}(Q) \equiv \rho(+-)$, called in the following the unlike-charge reference sample. This sample has the same topology and global properties as the like sign sample $\rho(++,--)$, but is free of any BEC effect. However, this sample contains hadron-pairs from the decay of resonances such as $\rho, \eta, \eta^{\prime}, \omega, \phi, K^{*}$, which are not present in the like-sign combinations. To account for the effects of resonances, the two-particle correlation function $C_{2}(Q)$ is corrected using Monte Carlo (MC) simulation without BEC effects via a double-ratio $R_{2}(Q)$ defined as

$$
R_{2}^{\mathrm{DR}}(Q)=\frac{C_{2}^{\text {Data }}(Q)}{C_{2}^{\mathrm{MC}}(Q)} .
$$

Various parameterizations of the $\Omega(\lambda, Q R)$ function can be found in the literature [3], each assuming a different shape for the particle-emitting source (Gaussian or exponential source, quantum optics model in Gaussian or exponential form, etc.).

In our analysis is used the following parameterizations:

- the Goldhaber parameterization of a static Gaussian source in the plane-wave approach, which assumes a spherical shape with a radial Gaussian distribution of the emitter

$$
C_{2}^{(G)}=C_{0}\left(1+\lambda \mathrm{e}^{-R^{2} Q^{2}}\right) \cdot(1+Q \varepsilon)
$$

- the exponential parameterization of a static source, which assumes a radial Lorentzian distribution of the source. This parameterization provides a better description of the data at small $Q$ values

$$
C_{2}^{(E)}=C_{0}\left(1+\lambda \mathrm{e}^{-R Q}\right) \cdot(1+Q \varepsilon)
$$

The first moment of the $\Omega(Q R)$ distribution corresponds to $1 / R$ for the exponential form and to $1 /(R \sqrt{ } \pi)$ for the Gaussian form. To compare the values of the radii obtained from the two functions, the $R$ value of the Gaussian should be compared to $R /{ } \pi$ of the exponential form.

\subsection{Data and MC samples}

The study is carried out using the minimum-bias ATLAS $p p$-collision datasets at the centre-of-mass energies $\sqrt{ }_{s}=0.9$ and $7 \mathrm{TeV}$ [4]. The event and track selection criteria are the same as the ones used for the ATLAS minimum-bias multiplicity analysis [4] with the same minimum-bias trigger and quality criteria for the track reconstruction.

All events in these datasets are required to have at least one vertex, formed from a minimum of two tracks with $p_{\mathrm{T}}>100 \mathrm{MeV}$ and consistent with the average beam spot position within the ATLAS detector [5]. Events with more than one primary vertex are rejected in order to prevent a bias from multiple proton-proton interactions (pile-up) in the colliding proton bunches. For the measurements at $\sqrt{s}_{s}=0.9 \mathrm{TeV}$, about $3.6 \times 10^{5}$ events with a total of more than $4.5 \times 10^{6}$ tracks are selected, and in the case of $\sqrt{s}_{s}=7 \mathrm{TeV}$, about $10^{7}$ events with about $2.1 \times 10^{8}$ tracks pass selection. This corresponds to integrated luminosities of $\sim 7 \mu \mathrm{b}^{-1}$ and $\sim 190 \mathrm{mb}^{-1}$ at $0.9 \mathrm{TeV}$ and $7 \mathrm{TeV}$, respectively. For the measurements at $7 \mathrm{TeV}$ with the high-multiplicity track trigger, about $1.8 \times 10^{4}$ events with more than $2.7 \times 10^{6}$ tracks overall were selected. This corresponds to integrated luminosity of $\sim 12.4 \mathrm{nb}^{-1}$. Large MC samples of minimum-bias events were generated using the PYTHIA 6.421 MC event generator [11] with the ATLAS 
MC09 set of optimized parameters [6] with non-diffractive, single-diffractive and doublediffractive processes. No simulation of the BEC effect has been implemented in the generator.

\subsection{Applied corrections}

The constructed two-particle density distributions for the data and MC samples are corrected for different detector inefficiencies and for some physics effects as is described below.

\section{3. 1 Coulomb correction}

The long-range Coulomb force causes a momentum shift between the like-sign and unlikesign pairs of particles. The density distributions are corrected for this effect using the Gamow penetration factor [7], $\quad N_{\text {meas }}(Q)=G(Q) N(Q) \quad$, where the Gamow factor $G(Q)$ is given by

$$
G(Q)=\frac{2 \pi \zeta}{\mathrm{e}^{2 \pi \zeta}-1} \quad ; \quad \zeta= \pm \frac{\alpha m}{Q}
$$

Here $\alpha=1 / 137$ is the electromagnetic fine-structure constant and $m$ is the particle mass. In the following the pion mass is considered. The sign of $\zeta$ is positive for like-sign pairs and negative for unlike-sign pairs.

The size of this correction is found not to exceed $20 \%$ for $Q>0.03 \mathrm{GeV}$. Neither the Coulomb interaction nor the BEC effect are present in the generation of $\mathrm{MC}$ event samples which are used in the analysis. The Coulomb correction is thus not applied to MC events.

\subsection{Event corrections}

The following corrections were taken into account in our analysis: - the trigger efficiency, $\varepsilon_{\text {trig }}(\mathrm{n})$, - the vertex reconstruction efficiency, $\varepsilon_{\text {vert }}(\mathrm{n})$, - unfolding to particle level, - pile-up effect fraction for high multiplicity events, - the track reconstruction efficiency, $\varepsilon\left(p_{t}, \eta\right)$, - the fraction of secondary particles, $f_{\sec }\left(p_{t}, \eta\right)$, - the fraction of selected tracks for which the corresponding primary particles are outside the kinematic range, $f_{\text {okr }}\left(p_{t}, \eta\right)$, - the fake tracks, $f_{\text {fake }}\left(p_{t}, \eta\right)$.

Each track was assigned a weight which corrects for the track reconstruction efficiency, for the fraction of secondary particles, for the fraction of the primary particles outside the kinematic range and for the fraction of fake tracks. The effect of events lost due to trigger and vertex reconstruction in efficiencies is corrected for using an event-by-event weight applied to pair of particles in the $Q$ distribution. The multiplicity distributions are corrected to the particle level using an iterative method that follows the Bayesian approach as it is described in Refs. [5]. An unfolding matrix, reflecting the probability of reconstructing $n_{\text {sel }}$ charged tracks in an event with generated charged particle multiplicity $n_{\mathrm{ch}}$ is populated using MC simulation and applied to the data. The unfolding matrix is built using the ATLAS MC09 PYTHIA tune. For all distributions, closure tests are carried out using MC samples corrected according to the same procedure as used in the data. The difference between the reweighted distributions and those at the particle level is due to tracking effects such as a smaller reconstruction efficiency for track pairs with very small opening angle. These effects are small for correlation functions constructed using data, typically $1-3 \%$, and are included in the systematic uncertainty. 


\subsection{Systematic uncertainties}

The systematic uncertainties on $\rho(Q)$ due to track reconstruction efficiency and track splitting and merging have been estimated to be $2-4 \%$ in the measured range of $Q$ from 0.02 $\mathrm{GeV}$ to $10 \mathrm{GeV}$. The leading source of systematic uncertainty is due to differences in the MC generators used to calculate the $\mathrm{R}_{2}$ correlation function from the $C_{2}$ correlation function. The corresponding contribution to the systematic uncertainty is estimated as the root-mean-squared (RMS) spread of the results obtained for the different MC datasets.

\section{Results}

In Fig. 1 the double-ratio $R_{2}(Q)$ distributions, measured for 0.9 and $7 \mathrm{TeV}$, are compared to the Gaussian and exponential parametrizations. The fits are performed in the $Q$ range from 0.02 $\mathrm{GeV}$ to $2 \mathrm{GeV}$ and with a bin width of $0.02 \mathrm{GeV}$. The upper $Q$ limit is chosen to be far away from the low- $Q$ region, which is sensitive to BEC effects and resonances. Around $Q \sim 0.7 \mathrm{GeV}$ there is a visible bump which is due to an overestimate of $\rho \rightarrow \pi+\pi^{-}$decays in the MC simulation. Therefore, the region $0.5 \leq Q \leq 0.9 \mathrm{GeV}$ is excluded from the fits. As is seen in Fig. 1, the Gaussian function does not describe the low- $Q$ region while the exponential fit provides a good description of the data. The BEC parameters obtained from fits of the two-particle doubleratio correlation function $R_{2}(Q)$ using the unlike-charge reference sample were found to be:

$$
\begin{aligned}
& \lambda=0.74 \pm 0.11, R=1.83 \pm 0.25 \text { at } \sqrt{s}=0.9 \mathrm{TeV} \text { for } n_{\mathrm{ch}} \geq 2, \\
& \lambda=0.71 \pm 0.07, R=2.06 \pm 0.22 \text { at } \sqrt{s}=7 \mathrm{TeV} \text { for } n_{\mathrm{ch}} \geq 2, \\
& \lambda=0.52 \pm 0.06, R=2.36 \pm 0.30 \text { at } \sqrt{s}=7 \mathrm{TeV} \text { for } n_{\mathrm{ch}} \geq 150 .
\end{aligned}
$$

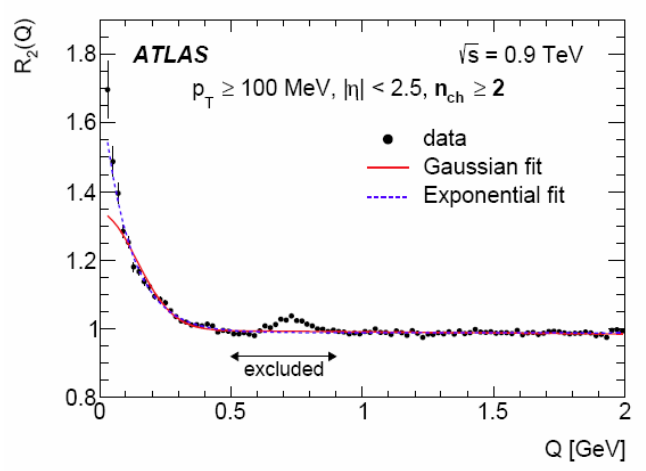

(a)

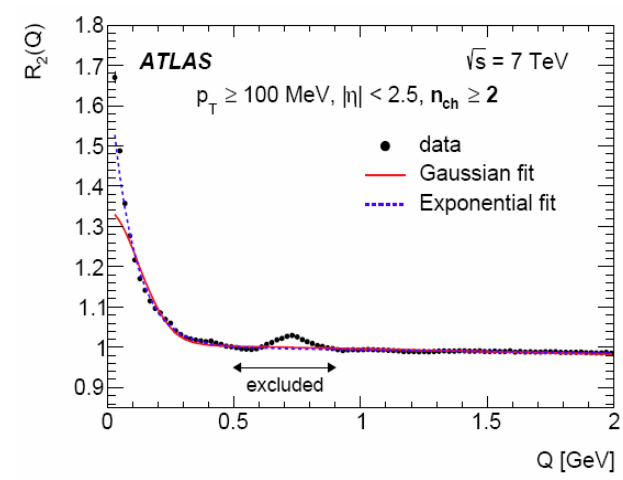

(b)

Fig. 1. The two-particle double-ratio correlation function, $R_{2}(Q)$, for charged particles in $p p$ collisions at $\sqrt{s}_{s}=0.9 \mathrm{TeV}$ (a), $7 \mathrm{TeV}$ (b) using the unlike-charge reference sample [1]. The region excluded from the fits is indicated. The error bars represent the statistical uncertainties.

\subsection{Multiplicity dependence}

The parameters from fitting of $R_{2}(Q)$ functions, are shown for various multiplicity intervals in Fig. 2 for $0.9 \mathrm{TeV}, 7 \mathrm{TeV}$ and $7 \mathrm{TeV}$ high-multiplicity data. The multiplicity intervals are chosen to be similarly populated and comparable to those used by other LHC experiments. Only the exponential fit is shown. As in the fit procedure for the inclusive case, the detector $Q$ resolution is included in the fits. The saturation of $R$ at high multiplicities is expected in a 
Pomeron-based model [8] as the consequence of the overlap of colliding protons, with the value of the radius at $n_{\mathrm{ch}} \approx 70$ close to the one obtained in the present studies. However, the same model predicts that above $n_{\mathrm{ch}} \approx 70, R$ will decrease with multiplicity, returning to its lowmultiplicity value clearly in contradiction with the measurement.

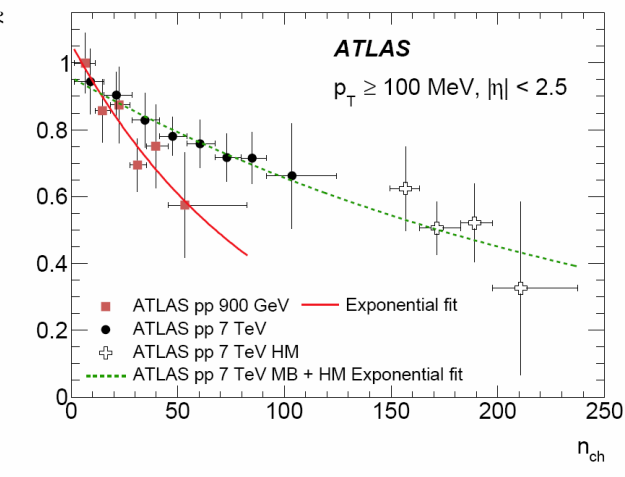

(a)

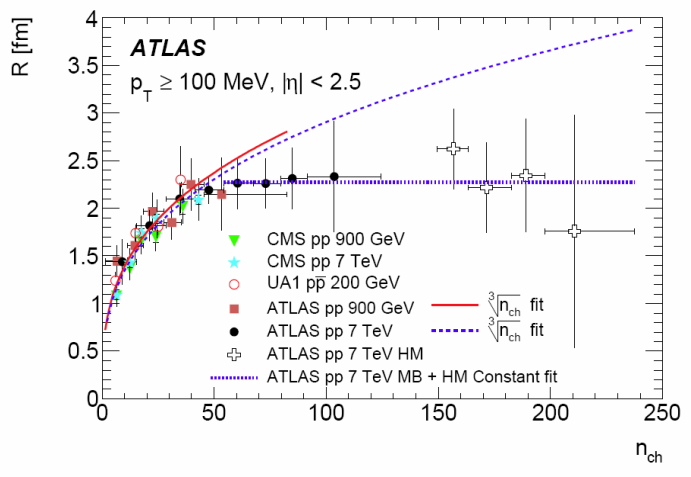

(b)

Fig. 2 Multiplicity, $n_{\mathrm{ch}}$, dependence of the $\lambda$ (a) and $R$ (b) parameters from the exponential fit to the $R_{2}(Q)$ at $\sqrt{ }_{s}=0.9$ and $7 \mathrm{TeV}$ [1], compared to the equivalent measurements of the CMS [9] and UA1 [10] experiments. The solid and dashed curves are the results of the exponential (a) and $3 \sqrt{ } n_{\mathrm{ch}}$ (b) for $n_{\mathrm{ch}}<55$ fits. The dotted line in (b) is a result of a constant fit to minimum-bias and high-multiplicity events data at $7 \mathrm{TeV}$ for $n_{\mathrm{ch}} \geq 55$. The error bars represent the quadratic sum of the statistical and systematic uncertainties.

The $\lambda$ parameter decreases with multiplicity, faster for $0.9 \mathrm{TeV}$ than for $7 \mathrm{TeV}$ interactions. The decrease of the $\lambda$ parameter with $n_{\mathrm{ch}}$ is found to be well fitted to the exponential function $\lambda\left(n_{\mathrm{ch}}\right)$ $=p_{0} \exp \left(-p_{1} n_{\mathrm{ch}}\right)$. The $R$ parameter increases with multiplicity up to about $n_{\mathrm{ch}} \sim 50$ independently of the center of mass energy. For higher multiplicities the $R$ parameter is independent of multiplicity. For $n_{\mathrm{ch}} \leq 82$ at $0.9 \mathrm{TeV}$ and $n_{\mathrm{ch}}<55$ at $7 \mathrm{TeV}$ the $n_{\mathrm{ch}}$ dependence of $R$ is fitted to the function $R\left(n_{\mathrm{ch}}\right)=p_{0} 3 \sqrt{ } n_{\mathrm{ch}}$.

\subsection{The $\mathrm{k}_{\mathrm{T}}$ dependence}

The average transverse momentum $k_{\mathrm{T}}$ of a particle pair is defined as half of the magnitude of the vector sum of the two transverse momenta, $k_{\mathrm{T}}=\left|\mathbf{p}_{\mathrm{T} ; 1}+\mathbf{p}_{\mathrm{T} ; 2}\right| / 2$.

The values of both $\lambda$ and $R$ decrease with increasing $k_{\mathrm{T}}$. The decrease of $\lambda$ with $k_{\mathrm{T}}$ is well described by an exponential function, $\lambda\left(k_{\mathrm{T}}\right)=p_{0} \exp \left(-p_{1} k_{\mathrm{T}}\right)$. The $k_{\mathrm{T}}$ dependence of the $R$ parameter is also found to follow an exponential decrease, $R\left(k_{\mathrm{T}}\right)=p_{0} \exp \left(-p_{1} k_{\mathrm{T}}\right)$. The shapes of the $k_{\mathrm{T}}$ dependence are similar for the $7 \mathrm{TeV}$ and the $7 \mathrm{TeV}$ high-multiplicity data. In Fig. 3, the $k_{\mathrm{T}}$ dependence of $\lambda$ and $R$, obtained for the $7 \mathrm{TeV}$ data, is also shown for various multiplicity regions: $2 \leq n_{\mathrm{ch}} \leq 9 ; 10 \leq n_{\mathrm{ch}} \leq 24 ; 25 \leq n_{\mathrm{ch}} \leq 80$; and $81 \leq n_{\mathrm{ch}} \leq 125$. The decrease of $\lambda$ with $k_{\mathrm{T}}$ is nearly independent of multiplicity for $n_{\mathrm{ch}}>9$ and the same as for the inclusive case. For $n_{\mathrm{ch}} \leq 9$ no conclusions can be drawn due to large uncertainties. The $R$-parameter decreases with $k_{\mathrm{T}}$ and exhibits an increase with multiplicity as observed for the fully inclusive case. 


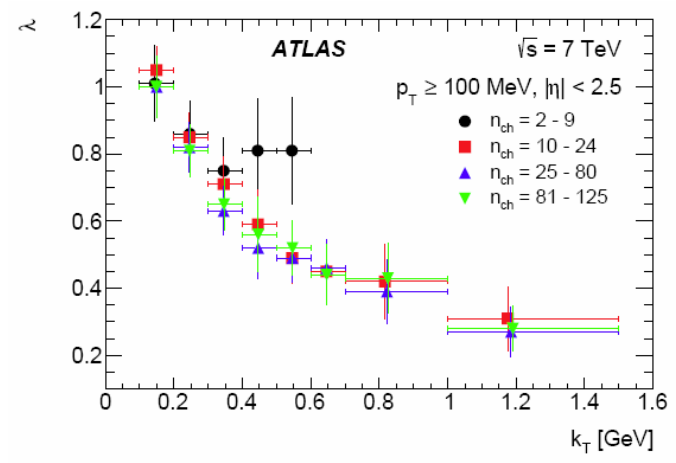

(a)

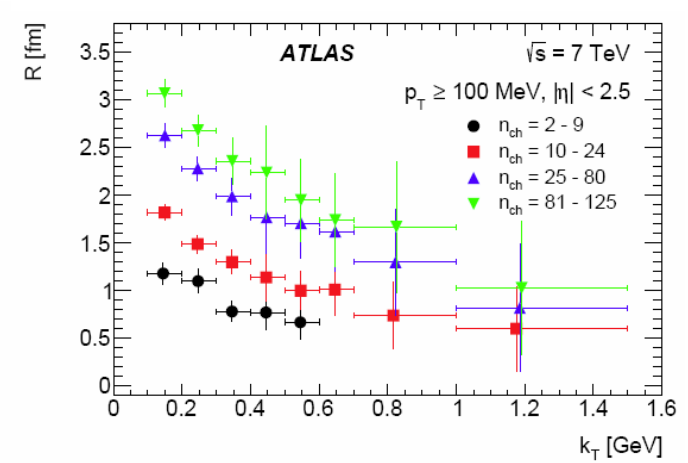

(b)

Fig. 3. The $k_{\mathrm{T}}$ dependence of the parameters $\lambda$ (a) and $R$ (b) from the exponential fit to the twoparticle double-ratio correlation function $R_{2}(Q)$ at $\sqrt{ }_{s}=7 \mathrm{TeV}$ with the unlike-charge reference sample for the different multiplicity regions [1].

\section{Conclusion}

The Bose-Einstein correlations of the pairs of identical charged particles have been measured in $|\eta|<2.5$ and $p_{\mathrm{T}}>100 \mathrm{MeV}$ in $p p$ collisions at 0.9 and $7 \mathrm{TeV}$ with the ATLAS detector at the LHC. A clear signal of BEC enhancement was observed in the region of small Q. Multiplicity dependence of the BEC was investigated up to very high multiplicities (up to 240). A saturation effect in multiplicity dependence of the extracted BEC radius was observed at level $R=2.28 \pm 0.32 \mathrm{fm}$. Dependence of the BEC parameters on track pair $k_{\mathrm{T}}$ and on particle $p_{\mathrm{T}}$ was investigated, too. A comparison with the measurements by other experiments at the same and lower energies was made. The measurements presented here complement the earlier measurements by extending the studies to higher multiplicities and transverse momenta. This provides a first observation of the saturation effect at high multiplicities of charged particles and confirms the exponential decrease of the radii with pair transverse momenta.

\section{References}

1. Atlas Collaboration, [arXiv:1502.07947] [hep-ex] 27 Feb 2015

2. ATLAS Collaboration, JINST 3, S08003 (2008)

3. W. Kittel and E.A. De Wolf, Soft Multihadron Dynamics (World Scientific, Singapore, 2005)

4. ATLAS Collaboration, New J. Phys. 13, 053033 (2011)[arXiv:1012.5104]

5. ATLAS Collaboration, J. High Energy Phys. 11, 033 (2012) [arXiv:1208.6256]

6. ATLAS Collaboration, Phys. Lett. B 688, 21 (2010) [arXiv:1003.3124]

7. M. Deutschmann et al., Nucl. Phys. B 204, 333 (1982)

8. V. A. Schegelsky, A. D. Martin, M. G. Ryskin and V. A. Khoze, Phys. Lett. B 703, 288 (2011)

9. CMS Collaboration, J. High Energy Phys. 05, 029 (2011) [arXiv:1101.3518]

10. C. Albajar et al. (UA1 Collaboration), Phys. Lett. B 226, 410 (1989)

11. T. Sjöstrand, S. Mrenna, P.Z. Skands, J. High Energy Phys. 05, 026 (2006) [hep-ph/0603175] 